\title{
Mechanism of Yinqin Oral Liquid in the Treatment of Chronic Pharyngitis Based on Network Pharmacology
}

\author{
Shiliang $\mathrm{Ji}^{\mathrm{I}}$ \\ Fei $X u^{2}$ \\ Ruifang Zhu' \\ Cheng Wang ${ }^{2,3}$ \\ Dongkai Guo' \\ Yiguo Jiang'
}

'Laboratory of Clinical Pharmacy, The Affiliated Suzhou Science and Technology Town Hospital of Nanjing Medical University, Suzhou, People's Republic of China; ${ }^{2}$ College of Pharmacy, Nanjing Medical University, Nanjing, People's

Republic of China; ${ }^{3}$ The Health Supervision Institute of Suzhou High-Tech Zone, Suzhou, People's Republic of China

Correspondence: Dongkai Guo; Yiguo Jiang Laboratory of Clinical Pharmacy, The Affiliated Suzhou Science and Technology Town Hospital of Nanjing Medical University, No. I Li Jiang Road, High-Tech District, Suzhou, 215153, People's Republic of China Tel +86-5 I2-69584330

Fax +86-5I 2-695845I3

Email dongkaiguo@126.com;

jiangyiguo0515@I26.com
Background: Yinqin oral liquid (YOL) has curative effect for upper respiratory tract infections, especially for chronic pharyngitis (CP). Since the traditional Chinese herbal formulae are complicated, the pharmacological mechanism of YOL remains unclear. The aim of this work was to explore the active ingredients and mechanisms of YOL against CP. Methods: First, the profile of putative target of YOL was predicted based on structural and functional similarities of all available YOL components, which were obtained from the Drug Bank database, to the known drugs using TCMSP. The chemical constituents and targets of honeysuckle, scutellaria, bupleurum and cicada were searched by TCMSP, CTD, GeneCards and other databases were used to query the CP-related genes, which were searched by UniProt database. Thereafter, the interactions network between compounds and overlapping genes was constructed, visualized, and analyzed by Cytoscape software. Finally, pathway enrichment analysis of overlapping genes was carried out on Database for Annotation, Visualization, and Integrated Discovery (DAVID) platform.

Results: The pathway enrichment analysis showed 55 compounds and 113 corresponding targets in the compound-target network, and the key targets involved PTGS1, ESR2, GSK3 $\beta$, NCOA2, ESR1. The PPI core network contained 30 proteins, including VEGFA, IL6, ESR1, RELA and HIF1A. A total of $148 \mathrm{GO}$ items were obtained $(p<0.05), 102$ entries on biological process (BP), 34 entries on biological process (BP) and 12 entries on cell composition (CC) were included. A total of 46 signaling pathways were obtained by KEGG pathway enrichment screening $(p<0.05)$, involving cancer, PI3K-AKT, hepatitis, proteoglycans, p53, HIF-1 signaling pathways.

Conclusion: These results collectively indicate YOL (including the main ingredients luteolin and baicalein) as a highly effective therapeutic agent for anti-inflammation, through the NF-kB pathway.

Keywords: Yinqin oral liquid, chronic pharyngitis, network pharmacology, NF-kB, multitarget analysis

\section{Introduction}

Chronic pharyngitis (CP) is a very common disease associated with chronic inflammation involving pharyngeal mucosa, submucosal and lymphatic tissues. Clinically, $\mathrm{CP}$ is mainly manifested as pharyngeal discomfort (foreign body sensation, burning sensation, irritation, etc.), with occasional pharyngeal itch, cough, etc., which belongs to the category of "slow throat arthralgia" in the traditional Chinese medicine (TCM). ${ }^{1} \mathrm{CP}$ has a high incidence and accounts for $10-12 \%$ of all pharyngeal diseases, with a long disease course, recurrence and is difficult to 
cure. At present, western medicine uses antibiotics supplemented by hormone preparations, such as dexamethasone and antiviral drugs, for the treatment of pharyngitis. These treatment methods have drawbacks such as narrow therapeutic spectrum, recurrence, poor tolerance and major toxic and side effects. Hence, developing more effective therapeutic strategies against $\mathrm{CP}$, and reducing the occurrence of side effects from the current therapeutic options is of great clinical importance.

TCM is a major component of complementary and alternative medicine. Owing to its excellent clinical efficacy, TCM is a research hotspot worldwide. Dialectical theory of TCM is used to treat acute and chronic pharyngitis and has unique advantages such as minor toxic side effects, obvious curative effect and treatment of both symptoms and root causes. ${ }^{2-4}$ The pharmacological effects of TCM herbal formulae play an important role in their appropriate application. However, the complex nature of herbal formulae has impeded this understanding. Numerous chemical ingredients involving multiple potential targets are present in an herbal formula. It is not adequate to explain the effects produced by a whole herbal formula if we individually consider every single ingredient in it. Yinqin oral liquid (YOL) is composed of extracts of honeysuckle, scutellaria, bupleurum and cicada. YOL has the effects of relieving wind, muscle and clearing heat, which is mainly used for cold, fever, aversion to cold, pharyngeal pain and other upper respiratory tract infections caused by wind fever and wind-cold. Long-term clinical observation has proven its effect in relieving pharyngeal pain and treating hand-foot-mouth disease. YOL contains several chemical compounds, which regulate diverse targets, and thus precisely determine the pharmacological mechanisms involved in its therapeutic actions. In addition, there are several challenges in the relationships between the herbs and diseases. ${ }^{5,6}$

Network pharmacology is based on the theory of system biology. As a new subject, it selects specific signal nodes to design multi-target drug molecules through the network analysis of system biology. It is possible to study both the active components and the potential gene targets from TCM because of the establishment of network pharmacology and bioinformatics. This study predicted and analyzed the active components and target of YOL using network pharmacology, to explore the rationality of its formula and the scientific nature of treating $\mathrm{CP}$, and further explore the pharmacological mechanisms of YOL on CP.

\section{Materials and Methods}

\section{Chemicals and Reagents}

Yinqin Oral Liquid (YOL) was provided by su zhou si yuan natural products research and development Co. Ltd. YOL is composed of honeysuckle, scutellaria, bupleurum and cicada, decompressed and concentrated to $2 \mathrm{~g} / \mathrm{mL}$ through boiling and alcohol sinking, and stored at $4^{\circ} \mathrm{C}$ for later use. Primary antibodies: COX-2 and iNOS antibodies were purchased from Abcam (Cambridge, UK); glyceraldehyde-3-phosphate dehydrogenase (GAPDH) antibody was obtained from Millipore (Billerica, MA, USA); PI3K, p-AKT, AKT, p-Stats, Stat3 and p-p65 (S536) antibodies were purchased from Cell Signaling Technology (Danvers, MA, USA); and p65 antibodies was obtained from Santa Cruz (CA, USA). The horseradish peroxidase (HRP)-conjugated sheep antimouse or anti-rabbit secondary antibodies were purchased from Thermo Fisher (Waltham, MA, USA). The proteins were visualized using an ECL detection kit (Thermo Fisher).

\section{Cell Culture}

RAW264.7 cells, a mouse macrophage cell line (Cell Bank of Chinese Academy of Sciences, Shanghai, China) were cultured using DMEM supplemented with FBS (10\%) and penicillin-streptomycin $(1 \%)$. The cells were cultured at $37^{\circ} \mathrm{C}$ in a humidified environment of $5 \%$ carbon dioxide. The medium was changed every other day and the cells were passaged at a dilution of 1:3.

\section{Collection of Drug Molecular Information and Screening of Active Ingredients}

In the TCMSP database (https://tcmsp-e.com/), ${ }^{7}$ the "Herb name" was selected to retrieve the molecular ADME parameter information of honeysuckle, scutellaria, bupleurum and cicada slough, and then the screen conformed to the oral bioavailability (OB) ADME parameter information of honey (Drug-likeness, DL) of $\geq 0.18$ or more active ingredients (Table 1). YOL ingredients were supplemented through literature review. Potential targets related to active ingredients were searched through the TCMSP database and Cytoscape 3.6.1 software.

\section{Known Therapeutic Targets of Chronic Pharyngitis (CP)}

In the drug treatment of $\mathrm{CP}$, the known therapeutic targets were mainly obtained from two sources: GeneCards database (https://www.genecards.org/) and CTD database (http://ctd.mdibl.org/). The data analysis was conducted 
Table I 55 Active Compounds Predicted by OB and DL Among 4 Herbs in YOL

\begin{tabular}{|c|c|c|c|c|c|}
\hline Drug & Mol ID & Molecule Name & MW & OB\% & DL \\
\hline Honeysuckle & $\begin{array}{l}\text { MOL0030I4 } \\
\text { MOL002773 } \\
\text { MOL003036 } \\
\text { MOL003044 } \\
\text { MOL003095 } \\
\text { MOL003III } \\
\text { MOL003II } \\
\text { MOL003I } 28 \\
\text { MOL000358 } \\
\text { MOL000422 } \\
\text { MOL000449 } \\
\text { MOL000006 } \\
\text { MOL000098 }\end{array}$ & $\begin{array}{l}\text { Mandenol } \\
\text { Ethyl linolenate } \\
\text { Eriodyctiol (flavanone) } \\
\text { (-)-(3R,8S,9R,9aS, I0aS)-9-ethenyl-8-(beta-D-glucopyranosyloxy)- } \\
\text { 2,3,9,9a, I0,I0a-hexahydro-5-oxo-5H,8H-pyrano[4,3-d]oxazolo } \\
\text { [3,2-a]pyridine-3-carboxylic acid_qt } \\
\text { Secologanic dibutylacetal_qt } \\
\text { Beta-carotene } \\
\text { ZINC0397878I } \\
\text { Chryseriol } \\
\text { 5-hydroxy-7-methoxy-2-(3,4,5-trimethoxyphenyl)chromone } \\
\text { Centauroside_qt } \\
\text { loniceracetalides B_qt } \\
\text { Dinethylsecologanoside } \\
\text { Beta-sitosterol } \\
\text { Kaempferol } \\
\text { Stigmasterol } \\
\text { Luteolin } \\
\text { Quercetin }\end{array}$ & $\begin{array}{l} \\
384.57 \\
536.96 \\
412.77 \\
300.28 \\
358.37 \\
434.48 \\
314.37 \\
434.44 \\
414.79 \\
286.25 \\
412.77 \\
286.25 \\
302.25\end{array}$ & $\begin{array}{l}53.65 \\
37.18 \\
43.83 \\
35.85 \\
51.96 \\
55.79 \\
61.19 \\
48.46 \\
36.91 \\
41.88 \\
43.83 \\
36.16 \\
46.43\end{array}$ & $\begin{array}{l}0.29 \\
0.58 \\
0.76 \\
0.27 \\
0.41 \\
0.5 \\
0.19 \\
0.48 \\
0.75 \\
0.24 \\
0.76 \\
0.25 \\
0.28\end{array}$ \\
\hline Scutellaria & $\begin{array}{l}\text { MOL000073 } \\
\text { MOL000I73 } \\
\text { MOL000228 } \\
\text { MOL000359 } \\
\text { MOL000525 } \\
\text { MOL000552 } \\
\text { MOL00I458 } \\
\text { MOL00I490 } \\
\text { MOL00I689 } \\
\text { MOL0027I4 } \\
\text { MOL002879 } \\
\text { MOL002897 } \\
\text { MOL002909 } \\
\text { MOL0029I0 } \\
\text { MOL0029I3 } \\
\text { MOL0029I4 } \\
\text { MOL0029I5 } \\
\text { MOL0029I7 } \\
\text { MOL002925 } \\
\text { MOL002927 } \\
\text { MOL002928 } \\
\text { MOL002932 } \\
\text { MOL002933 } \\
\text { MOL002934 } \\
\text { MOL002937 } \\
\text { MOL008206 } \\
\text { MOL0I04I5 } \\
\text { MOL0I2266 } \\
\text { MOL000490 } \\
\text { MOL004598 }\end{array}$ & $\begin{array}{l}\text { Ent-Epicatechin } \\
\text { Wogonin } \\
\text { (2R)-7-hydroxy-5-methoxy-2-phenylchroman-4-one } \\
\text { Sitosterol } \\
\text { Norwogonin } \\
\text { 5,2'-Dihydroxy-6,7,8-trimethoxyflavone } \\
\text { Coptisine } \\
\text { Bis[(2S)-2-ethylhexyl] benzene-I,2-dicarboxylate } \\
\text { Acacetin } \\
\text { Baicalein } \\
\text { Diop } \\
\text { Epiberberine } \\
\text { 5,7,2,5-tetrahydroxy-8,6-dimethoxyflavone } \\
\text { Carthamidin } \\
\text { Dihydrobaicalin_qt } \\
\text { Eriodyctiol (flavanone) } \\
\text { Salvigenin } \\
\text { 5,2',6'-Trihydroxy-7,8-dimethoxyflavone } \\
\text { 5,7,2',6'-Tetrahydroxyflavone } \\
\text { Skullcapflavone II } \\
\text { Oroxylin a } \\
\text { Panicolin } \\
5,7,4^{\prime} \text {-Trihydroxy-8-methoxyflavone } \\
\text { Neobaicalein } \\
\text { Dihydrooroxylin A } \\
\text { Moslosooflavone } \\
\text { II,I3-Eicosadienoic acid, methyl ester } \\
\text { Rivularin } \\
\text { Petunidin } \\
\text { 3,5,6,7-tetramethoxy-2-(3,4,5-trimethoxyphenyl)chromone }\end{array}$ & $\begin{array}{l}290.29 \\
284.28 \\
270.3 \\
414.79 \\
270.25 \\
344.34 \\
320.34 \\
390.62 \\
284.28 \\
270.25 \\
390.62 \\
336.39 \\
376.34 \\
288.27 \\
272.27 \\
288.27 \\
328.34 \\
330.31 \\
286.25 \\
374.37 \\
284.28 \\
314.31 \\
300.28 \\
374.37 \\
286.3 \\
298.31 \\
322.59 \\
344.34 \\
317.29 \\
432.46\end{array}$ & $\begin{array}{l}48.96 \\
30.68 \\
55.23 \\
36.91 \\
39.4 \\
31.71 \\
30.67 \\
43.59 \\
34.97 \\
33.52 \\
43.59 \\
43.09 \\
33.82 \\
41.15 \\
40.04 \\
41.35 \\
49.07 \\
45.05 \\
37.01 \\
69.51 \\
41.37 \\
76.26 \\
36.56 \\
104.3 \\
66.06 \\
44.09 \\
39.28 \\
37.94 \\
30.05 \\
31.97\end{array}$ & $\begin{array}{l}0.24 \\
0.23 \\
0.2 \\
0.75 \\
0.21 \\
0.35 \\
0.86 \\
0.35 \\
0.24 \\
0.21 \\
0.39 \\
0.78 \\
0.45 \\
0.24 \\
0.21 \\
0.24 \\
0.33 \\
0.33 \\
0.24 \\
0.44 \\
0.23 \\
0.29 \\
0.27 \\
0.44 \\
0.23 \\
0.25 \\
0.23 \\
0.37 \\
0.31 \\
0.59\end{array}$ \\
\hline
\end{tabular}

(Continued) 
Table I (Continued).

\begin{tabular}{|l|l|l|l|l|l|}
\hline Drug & Mol ID & Molecule Name & MW & OB\% & DL \\
\hline Bupleurum & MOL002776 & Baicalin & 446.39 & 40.12 & 0.75 \\
& MOL00I645 & Kaempferol & 308.56 & 42.1 & 0.2 \\
& MOL0047I8 & Linoleyl acetate & 412.77 & 42.98 & 0.76 \\
& MOL004653 & $\alpha$-spinasterol & 426.5 & 46.06 & 0.66 \\
& MOL004624 & Stigmasterol & 348.48 & 47.72 & 0.53 \\
& MOL004609 & Areapillin & 360.34 & 48.96 & 0.41 \\
& MOL000354 & Isorhamnetin & 316.28 & 49.6 & 0.31 \\
& MOL013I87 & Cubebin & 356.4 & 57.13 & 0.64 \\
\hline
\end{tabular}

in 5878 therapeutic targets for the treatment of $\mathrm{CP}$, after the redundant entries were removed. Figure S1 shows the specific information about these known therapeutic targets.

\section{Network Construction and Analysis}

To understand the relationship between the herbs and chemical compounds that consist of YOL and its putative targets, and the therapeutic targets known for CP, Network Visualization was conducted using Cytoscape 3.6.1 software, ${ }^{8}$ and the degree between compounds and targets was analyzed.

\section{Protein-Protein Interaction (PPI) Data}

PPI core network (PPICN) is used to study the relationship between chemical compounds and disease-associated protein molecules based on biochemistry, signal transduction and genetic networks. ${ }^{9}$ The differing ID types of the proteins were converted to UniProt IDs. In order to further understand how YOL and CP interact at the protein level, the selected targets in this study were uploaded on the online Venn diagram (http://bioinfogp.cnb.csic.es/tools/ venny/index.html). Moreover, the genes at the intersection of the active compound and CP were selected and uploaded on STRING 10.5 (https://string-db.org) to obtain the PPI relationship.

\section{Pathway Enrichment Analysis}

The data was imported in the format Gene Symbol into David 6.8 database (https://david.ncifcrf.gov/). Molecular function (CC), biological process (BP), and cellular function (MF) were selected, respectively. A pathway enrichment analysis was prospectively performed using biological process enrichment GO analysis and KEGG pathway analysis (http://www.genome.jp/kegg/) to clarify the pathways involving putative $\mathrm{CP}$ targets, and visualized by online mapping website Omicshare Tools (http://www. omicshare.com/tools/index.php/).

\section{Statistical Analysis}

Each experiment was repeated at least in triplicate and data were shown as mean \pm SD. Statistical difference in GO and KEGG analysis was performed using hypergeometric and Fisher's exact tests. $P<0.05$ was considered statistically significant.

\section{Results}

\section{Screening and Analysis of Active Compounds}

First, all the compounds related to four TCMs were retrieved by TCMSP. Second, according to the screening conditions of $\mathrm{OB} \geq 30 \%$ and $\mathrm{DL} \geq 0.18,{ }^{10} 23$ active constituents of honeysuckle (17 predicted relevant targets), 36 of baicalensis (30 predicted relevant targets), 17 of bupleurum (13 predicted relevant targets), and no suitable compounds of cicada slough were obtained. Among them, baicalensis and honeysuckle contained two identical ingredients, while bupleurum and honeysuckle contained three identical components. Finally, a total of 55 active components were obtained for the data analysis after removing redundant entries (Table 1).

\section{Network Analysis of "Active}

\section{Component-Target" Interaction in YOL}

The compound-target network contained 175 nodes including 55 compound nodes and 113 target nodes, of which two had no corresponding target and 577 edges. As shown in Figure 1, blue represents compounds in bupleurum, red represents compounds in scutellaria, purple represents compounds in honeysuckle, green represents drug targets, each edge indicates the interaction between compounds and their 


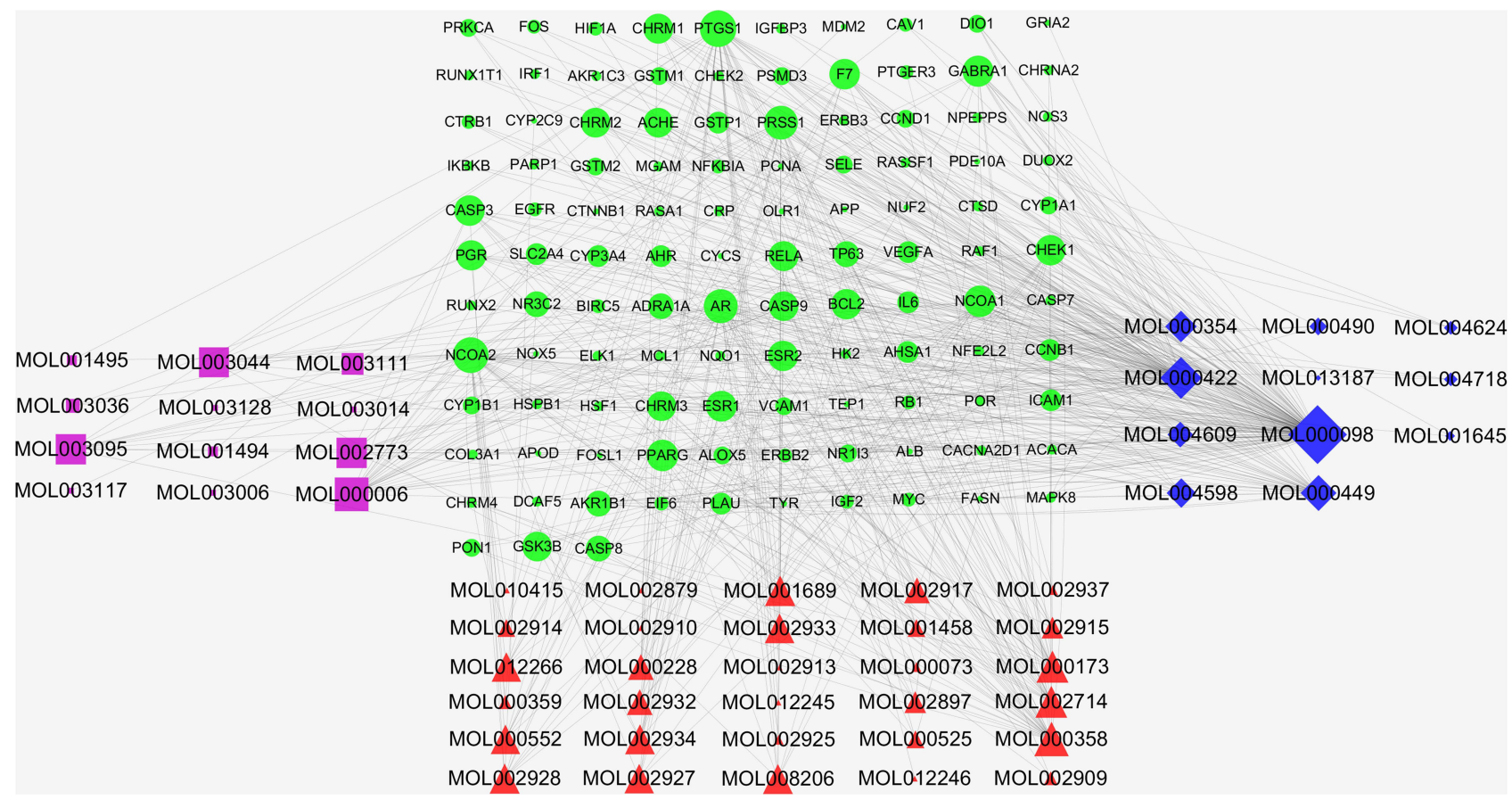

Figure I Compounds-target network diagram. Blue represents compounds in bupleurum, Red represents compounds in scutellaria, Purple represents compounds in honeysuckle, Green represents drug targets. The size represents the degree value. The larger the shape, the larger the degree value.

targets, and two of the 55 compounds were excluded from the network construction. The degree value of a node represents the amount of connected routes in the network, and the larger the shape, the higher the degree value. The network in line with its topological properties showed that the nodes with higher degree of screening were analyzed. These nodes that connect compounds or targets act as hubs in the whole network and may predict key compounds or targets. The top five compounds ranked by degree were MOL000098-quercetin, MOL000422-Kaempferol, MOL000449-stigmasterol, MOL 000358-quebrachol and MOL000006-luteolin, which could interact with 154, 68, 39, 32 and 28 target proteins, respectively. PTGS1, NCOA2, AR, PRSS1 and PPARG were the top five targets with the highest degree, which could interact with 42, 38, 29, 28 and 17 compounds, respectively.

\section{The Construction of Key Protein Network of YOL and CP}

Through the internationally recognized CTD and GeneCards disease databases, 5150 and 1247 pharyngitis-related targets were obtained, respectively. Through the Venny online tool, 30 targets of honeysuckle, scutellaria, bupleuri and pharyngitis were obtained, including VEGFA, IL6, ESR1, RELA, HIF1A, etc. (Figure 2A). Finally, STRING online tool was used to construct the PPI network interaction map of drug and disease. It contained 30 nodes representing proteins and
48 edges representing the interaction between proteins. The thicker the line, the higher is the correlation degree. Through the protein interaction network, we could further explore the therapeutic target and mechanism of YOL for $\mathrm{CP}$ (Figure 2B).

\section{Pharmacological Mechanisms of YOL Acting on CP}

A total of 30 common targets were obtained by DAVID online tool and $148 \mathrm{GO}$ items were obtained $(\mathrm{p}<0.05)$ by performing the functional enrichment analysis. There were 102 entries on biological process (BP), 34 entries on Molecular Function (MF), and 12 entries on cell composition (CC) (Figure 3A). A total of 46 signaling pathways were obtained by KEGG pathway enrichment screening $(p<0.05)$, involving cancer, PI3K-AKT, hepatitis, proteoglycans, p53, HIF-1 signaling pathways, etc. (Figure 3B and Table S1).

\section{Experimental Verification of Predicted Results}

In order to experimentally demonstrate the predictions about the molecular mechanism of YOL, we chose two ingredients for testing (Figure 4), based on their composition score, the group to which they belong, and their herbal origin to examine how ingredients from different herbs 

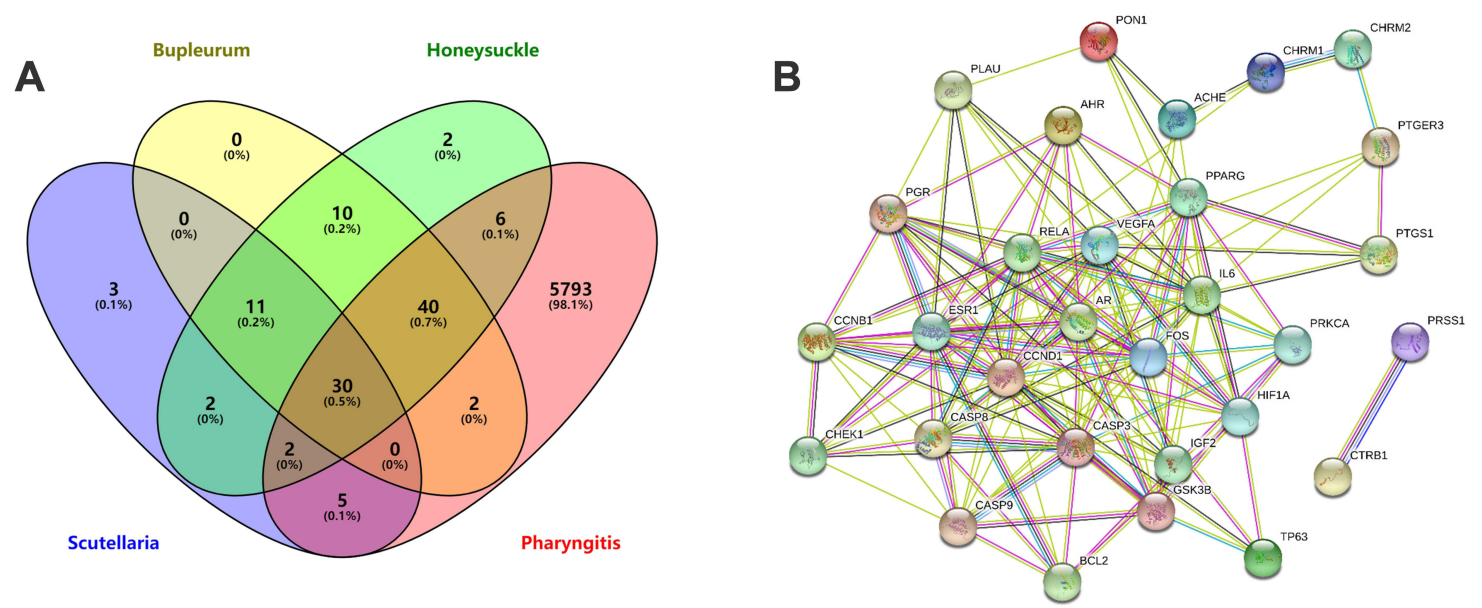

Figure 2 (A) Venn diagram of compounds-target network diagram; (B) Core network diagram of protein interaction between YOL and CP.

affect the same proteins. The effects of these compounds on the expression levels of related proteins were analyzed by Western blot. Macrophages secrete several inflammatory cytokines, which play significant roles in inflammation. Signal transduction and transcriptional activator 3 (STAT3), nuclear factor kappa B (NF-kB), and phosphatidylinositol 3-kinase (PI3K)/Akt pathways are critical inflammatory pathways. LPS can significantly increase the production of many proinflammatory factors, including inducible NO synthase (iNOS) and cyclooxygenase-2 (COX-2) in macrophages. After pretreatment of macrophages with YOL, luteolin and baicalein, the protein expression levels of iNOS and COX-2 induced by LPS were significantly inhibited in a dose-dependent manner (Figure 4A). The related protein changes were consistent with iNOS and COX-2 inflammation protein expression (Figure 4B-D).

\section{Discussion}

Many diseases, including cancer and chronic inflammation, are known to be regulated by multiple signaling pathways. TCMs are considered as multi-component and multi-target therapeutic drugs, which is in accordance with the methodologies of network pharmacology. The holistic view of TCM considers the human body as a complex biological network system, which is connected with network pharmacology. By combining the network pharmacology with the holistic view of TCM, the disease-target-drug network is obtained. The network shows that different components of TCM may act on one target,
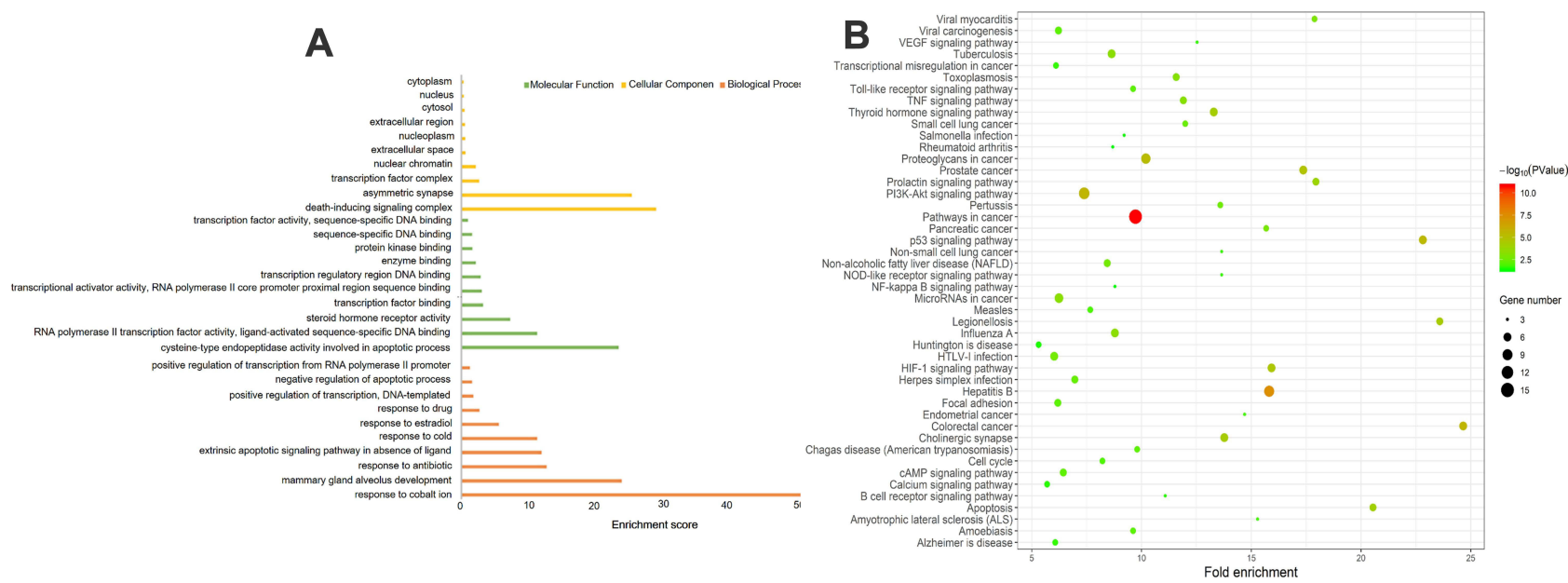

Figure 3 (A) GO analysis function annotation diagram. Biological process (BP), molecular function (MF), and cell composition (CC). (B) Enriched KEGG pathways of YOL selected targets for CP. 

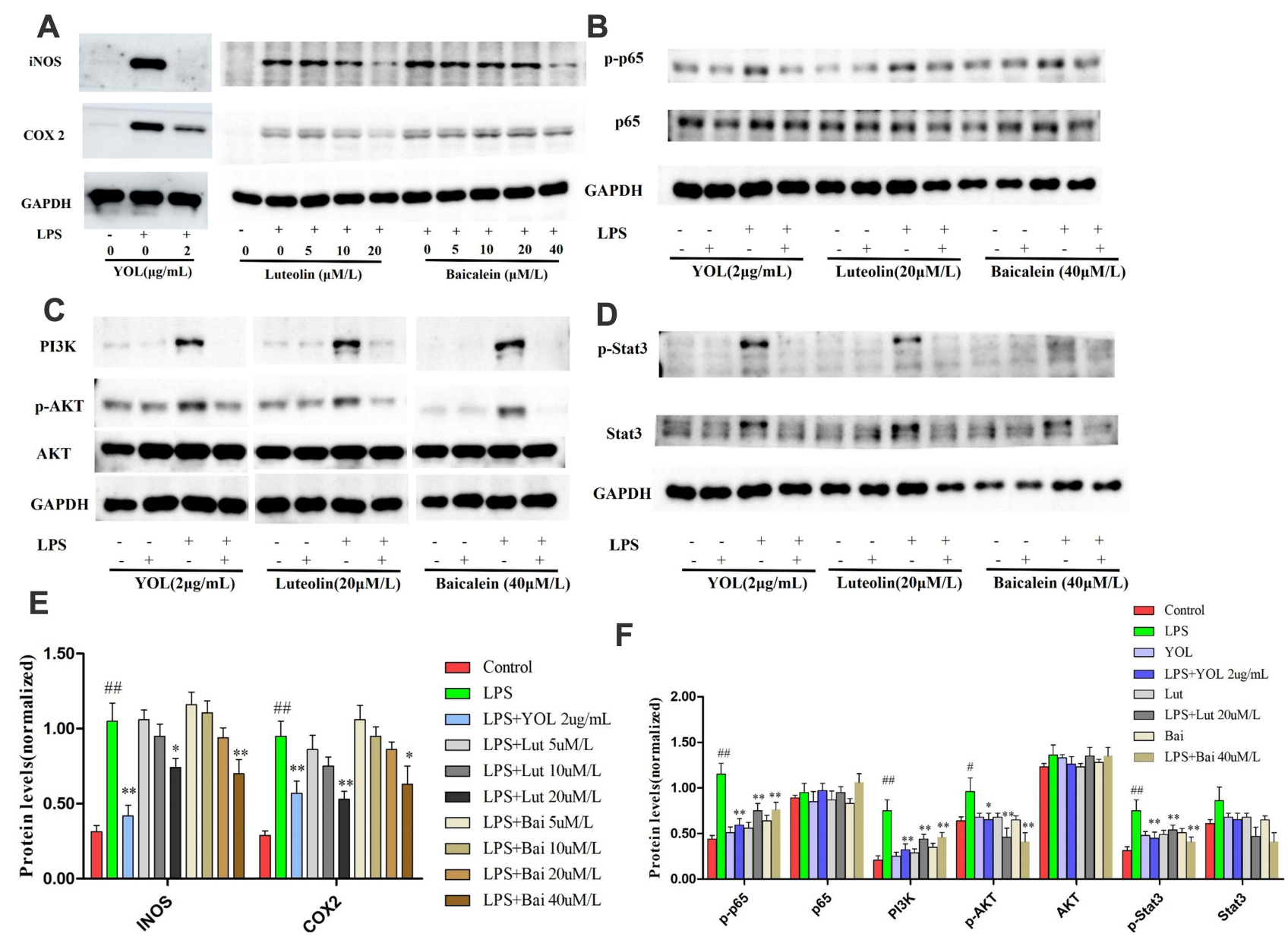

Figure 4 The YOL inhibits PI3K/p-AKT/p-Stats pathway in RAW 264.7 cells. Representative Western blot images show the relative expressions of iNOS, COX-2 (A), p-p65, p65 (B), PI3K, p-AKT, AKT (C), p-Stats, Stat3 (D) in the groups. The protein levels (normalized) of iNOS, COX2 (E), and p-p65, p65, PI3K, p-AKT, AKT, p-Stats, Stat3 (F) in cells. All the values are presented as mean \pm SD. ${ }^{\#} P<0.05$ and ${ }^{\# \#} P<0.01$ vs control group. ${ }^{*} P<0.05$ and ${ }^{* * P}<0.01$ vs LPS group.

and different targets may act on the same pathway. It is also possible that the same component of TCM acts on different targets, and the same target acts on different pathways. The multi-component, multi-target and multipathway of TCM can be clearly visualized through network pharmacology, which resolves many difficulties encountered in the study of TCM. ${ }^{13-15} \mathrm{CP}$ is the diffuse inflammation of pharyngeal mucosa, submucosa and lymph tissues. Clinically, $\mathrm{CP}$ is mainly manifested as pharyngeal discomfort (foreign body sensation, burning sensation, stimulation sensation, etc.), pharyngeal itching, cough, etc. Pharyngitis belongs to the category of "Fenghou Bi" in TCM. Pharyngitis pertains to the category of "wind throat arthralgia" in Chinese medicine, and is often caused by evil heat entering the body, and heat in the lungs and stomach. YOL is an oral TCM preparation, which is further optimized on the basis of Jinchan oral liquid developed by Children's Hospital of Suzhou
University. ${ }^{5,6}$ However, there is a lack of research on its active ingredients and its mechanism of action.

In this study, 30 potential targets, 102 biological processes, 12 molecular functions, 34 cell components, and 46 KEGG pathways were obtained. The active ingredients in YOL can treat CP through multiple targets and pathways. To elucidate the biological pathways that may be affected by YOL, a significantly overexpressed KEGG pathway was identified. The results showed VEGFA, IL6, ESR1 and RELA as the key targets and the involvement of PI3K-Akt, p53 and HIF-1 signaling pathways. TCM aims to restore a patient's health through the use of a TCM prescription, which is usually composed of two or more TCMs in optimal proportions.

YOL, for example, consists of four TCMs. As expected, most of the selected active ingredients are directly or indirectly associated with inflammation. In a previous study, neochlorogenic acid was demonstrated to inhibit LPS-activated 
inflammatory responses through up-regulation of Nrf2/HO-1 and AMPK pathways. ${ }^{16}$ The decoction extract from six natural herbs, which contain honeysuckle, exhibits antiinflammatory and immunomodulatory effects by targeting NF- $\mathrm{B} / \mathrm{IL}-6 / \mathrm{STAT} 3$ signaling. ${ }^{17}$ Luteolin is abundant in honeysuckle, which alleviates $\mathrm{CP}$ by inhibiting the NF- $\mathrm{BB}$ pathway and anti-inflammatory polarization of M1 macrophages. ${ }^{11}$ Protective effects of baicalein on liver injury in mice induced by multi-microbial septicemia were found to be based on inhibiting inflammation. ${ }^{12}$ Network pharmacological studies have shown that the main active components of honeysuckle may play a role through inflammation-related proteins such as HSP90AA1, HSP90AB1, ESR1, PTGS2, TERT and PPARG, and especially heat shock protein HSP90A (HSP90AA1). ${ }^{18}$ Flavonoids such as baicalin and wogonin alone and in combination have anti-inflammatory activity in Scutellaria. ${ }^{19}$ Network pharmacological studies have also shown that alkaloids such as coptisine and epiphone in Scutellaria and components such as dihydrokaempferol, and rographolide flavonoids also show antiinflammatory activity. MAPK14, TNFRSF1A, EGFR and SELE are the primary anti-inflammatory components of Scutellaria. The anti-inflammatory active components in Scutellaria can directly affect MAPK14 and EGFR to produce anti-inflammatory effects, and can also indirectly affect other targets to exert anti-inflammatory effects. As one of the four p38 MAPKs, MAPK14 plays a vital role in the cellular cascade induced by extracellular stimuli such as proinflammatory cytokines or physical stimuli. ${ }^{20}$ Studies predicted that the pharmacodynamic basis of Bupleurum is mainly saponins, flavonoids, volatile oils, fatty acids and other components. Flavonoids mainly act on PI3K-Akt, NF-kB and other pathways, participate in regulating inflammatory factors, estrogen signal transduction and other physiological processes. Bupleurum-Scutellaria drug pair, first used in small bupleurum decoction in Treatise on Febrile Diseases, is an important part of Bupleurum prescription. Bupleurum is bitter, flat and clear, and can disperse the stagnation of bile fire. Scutellaria has a bitter and cold taste, and can clear internal heat. Both of them must be used together to regulate the liver and gallbladder, and clear the dampness and heat of internal accumulation, which are commonly used to treat fever, pharyngitis, and respiratory diseases. ${ }^{21}$ Cicada slough can evacuate wind and heat, and clear the throat. Clinically, it is common in classical prescriptions such as Sheng jiang San and Pharyngitis San. However, the active components of Cicada slough did not satisfy the prediction requirements in this research.
YOL is composed of honeysuckle, scutellaria, bupleurum and cicada slough. According to TCM theory, bupleurum is bitter and flat, enters the liver and gallbladder meridian, penetrates and clears Shaoyang, and can drain the stagnation of gas. Scutellaria is bitter and cold, and clears the heat of Shaoyang. The actions of Bupleurum facilitate the clear discharge of Scutellaria, the two are compatible, and achieve the goal of reconciliation and Shaoyang. Honeysuckle cools through the surface, has heat-clearing and detoxifying effects, and also has the effect of fragrant obscenity. Cicada slough is light floating, clears the coke gas, shows honeysuckle compatibility, understanding the table evil, and is combined with the "treatment of the coke such as feather." The combination of the four drugs, Xuan and descent together, clear the evil, thereby clearing heat and detoxification, warming the evil without hiding, scattered outside and recovered. Therefore, the treatment of CP with YOL can control the pharyngitis, regulate the balance of Yin and Yang, strengthen the foundation and improve the immunity of the body.

\section{Conclusion}

This study used the network pharmacology platform to probe the YOL treatment of CP by multi-target analysis. The results showed that YOL could treat $\mathrm{CP}$ by acting on related targets, which were consistent with the reported literature. In addition, we have verified that YOL and its two monomer compounds, Luteolin and Baicalein inhibited activation of the NF-kB, Stat3 and PI3K-Akt pathways. Although the potential advantages of the "network target, multi-component" strategy of network pharmacology are obvious, the content of TCM ingredients is usually ignored in the research of network pharmacology of Chinese medicine, and the influence of content and concentration on the efficacy cannot be ignored. The predicted targets, which have not been discussed, may provide clues for further research on the mechanism of YOL.

\section{Funding}

This work was supported by the Third Batch of Suzhou High-tech District Health Talents Project SGXWS2020 (to Dr. Ji) and Suzhou Science and Technology Plan Project, (SYSD2019171, SYS2020076).

\section{Disclosure}

The authors report no conflicts of interest in this work. 


\section{References}

1. Li ZC, Huang JL, Hu ZP. Screening and diagnosis of chronic pharyngitis based on deep learning. Int $J$ Environ Res Public Health 2019;16(10):1688. doi:10.3390/ijerph16101688

2. Li CX, Wu FC, Yuan WL, et al. Systematic review of herbal tea (a traditional Chinese treatment method) in the therapy of chronic simple pharyngitis and preliminary exploration about its medication rules. Evid Based Complement Alternat Med. 2019:1-15. doi:10.1155/2019/9458676.

3. Qin LI, Rong HU, Zhao Y, et al. The studies on pharmacology of Jin xuan li yan granules against pharyngitis. Chin J Tradit Med. 2015;33 (5):1209-1211. doi:10.13193/j.issn.1673-7717.2015.05.058

4. Wu R, Wang ZF, Wei JP. TCM treatment for chronic pharyngitis. Inform J Tradit Chin Med. 2018;35(5):63-65. doi:10.19656/j. cnki.1002-2406.180150

5. Zhang L, M Q L, Dou J, et al. Studies on the anti-influenza virus activity of Jin chan oral solution in vitro and in vivo. Pharm Biotechnol. 2009;16(5):439-442. doi:10.19526/j.cnki.1005-8915.20 09.05.011

6. Shen L, Wang C, Hao CL, et al. Efficacy and safety of Jin-chan oral liquid in treatment of infants with respiratory syncytial virus pneumonia. Chin J New Drugs Clin Remedies. 2017;36(7):409-413. doi:10.14109/j.cnki.xyylc.2017.07.008

7. Ru JL, Li P, Wang JN, et al. TCMSP: a database of systems pharmacology for drug discovery from herbal medicines. $J$ Cheminform. 2014;6(1):1-6. doi:10.1186/1758-2946-6-13

8. Otasek D, Morris JH, Bouças J, Pico AR, Demchak B. Cytoscape automation: empowering workflow-based network analysis. Genome Biol. 2019;20(1):185. doi:10.1186/s13059-019-1758-4

9. Luo SW, Liang Z, Wu JR. Simultaneously measuring multiple protein interactions and their correlations in a cell by protein-interactome foot printing. Sci Rep. 2017;24(7):45169. doi:10.1038/srep45169

10. Zhang YQ, Mao X, Guo QY, et al. Network pharmacology-based approaches capture essence of Chinese herbal medicines. Chin Herb Med. 2016;8(2):107-116. doi:10.1016/S1674-6384(16)60018-7

11. Chen XM, Lai YF, Song XC, et al. Polysaccharides from Citrus grandis associate with luteolin relieves chronic pharyngitis by antiinflammatory via suppressing NF-kB pathway and the polarization of M1 macrophages. Int J Immunopathol Pharmacol. 2018;32:1-7. doi: $10.1177 / 2058738418780593$
12. Liu A, Wang WJ, Fang HS, et al. Baicalein protects against polymicrobial sepsis-induced liver injury via inhibition of inflammation and apoptosis in mice. Eur $J$ Pharmacol. 2015;5(748):45-53. doi:10.1016/j.ejphar.2014.12.014

13. Li L, Yang DL, Li JH, et al. Investigation of cardiovascular protective effect of Shenmai injection by network pharmacology and pharmacological evaluation. BMC Complement Med Ther. 2020;20 (1):330-352. doi:10.1186/s12906-020-02905-8

14. Cui SN, Chen SS, Qq W, et al. A network pharmacology approach to investigate the anti-inflammatory mechanism of effective ingredients from Salvia miltiorrhiza. Int Immunopharmacol. 2020;81:106040. doi:10.1016/j.intimp.2019.106040

15. Gu S, Pei JF. Chinese herbal medicine meets biological networks of complex diseases: a computational perspective. Evid Based Complement Alternat Med. 2017;2017:7198645. doi:10.1155/2017/ 7198645

16. Park SY, Jin ML, Yi EH, et al. Neochlorogenic acid inhibits against LPS-activated inflammatory responses through up-regulation of Nrf2/ HO-1 and involving AMPK pathway. Environ Toxicol Pharmacol. 2018;62:1-10. doi:10.1016/j.etap.2018.06.001

17. Lu X, Wo GQ, Li BH, et al. The anti-inflammatory NHE-06 restores antitumor immunity by targeting NF-kB/IL-6/STAT3 signaling in hepatocellular carcinoma. Biomed Pharmacother. 2018;102:42 0-427. doi:10.1016/j.biopha.2018.03.099

18. Du YQ, Duan ZK, Dong SH, et al. Anti-inflammatory mechanism of honeysuckle active ingredient based on network pharmacology. Chin $J$ Med Chem. 2019;29(2):96-102. doi:10.14142/j.cnki.cn21-1313/ r.2019.02.002

19. Feng T, Zhou LY, Sc G, et al. Acacia catechu (L.f.) Willd and Scutellaria baicalensis Georgi extracts suppress LPS-induced proinflammatory responses through NF-KB, MAPK, and PI3K-Akt signaling pathways in alveolar epithelial type II cells. Phytother Res. 2019;33(12):3251-3260. doi:10.1002/ptr.6499

20. Lei QL, Huang YL, Zhong Q, et al. Anti-inflammatory mechanism of Scutellarlae Radix based on network pharmacology. Chin Tradit Herb Drugs. 2018;49(15):3523-3530. doi:10.7501/j.issn.02532670.2017.15.009

21. Shen $\mathrm{X}$, Zhao ZY, Wang $\mathrm{H}$, et al. Elucidation of the anti-inflammatory mechanisms of Bupleuri and Scutellariae radix using system pharmacological analyses. Mediators Inflamm. 2017;2017:3709874. doi:10.1155/2017/3709874.

\section{Publish your work in this journal}

Drug Design, Development and Therapy is an international, peerreviewed open-access journal that spans the spectrum of drug design and development through to clinical applications. Clinical outcomes, patient safety, and programs for the development and effective, safe, and sustained use of medicines are a feature of the journal, which has also been accepted for indexing on PubMed Central. The manuscript management system is completely online and includes a very quick and fair peer-review system, which is all easy to use. Visit http://www. dovepress.com/testimonials.php to read real quotes from published authors. 